\title{
EFECTOS DE LA CRISIS EN LA ESTRUCTURA OCUPACIONAL Y LA BIOGRAFIA LABORAL DE LA POBLACIÓN INMIGRANTE ${ }^{1}$
}

\section{EFFECTS OF THE CRISIS ON THE OCCUPATIONAL STRUCTURE AND WORK BIOGRAPHY OF IMMIGRANT POPULATION}

Concepción Carrasco Carpio* y Carlos García-Serrano**

Resumen: Este artículo tiene como objetivo profundizar en el estudio del cambio ocupacional y de las trayectorias laborales de los individuos a lo largo del tiempo para ofrecer una visión más amplia del efecto de la crisis económica de 2008-2013 sobre el empleo. Para ello se utilizan los microdatos de la Encuesta de Población Activa y se realiza una comparación entre tres colectivos: españoles nacidos en España, españoles nacidos fuera de España. y extranjeros nacidos fuera de España. Los resultados avalan para el colectivo de extranjeros nacidos fuera la existencia de una estructura ocupacional segmentada hacia puestos de naturaleza manual (tanto cualificada como no cualificada), hecho que ha potenciado una pérdida de ocupación en la biografía laboral de las cohortes de individuos más jóvenes en el periodo recesivo.

Profesor Contratado Doctor, Universidad de Alcalá.

** Profesor Titular de Universidad, Universidad de Alcalá

1 Este artículo se ha desarrollado en el marco de dos proyectos de investigación: a) el proyecto $\mathrm{I}+\mathrm{D}+\mathrm{i}$ «Género,transnacionalismo y estrategias intergeneracionales de movilidad social» (FEM2011-26210); b) el proyecto "Inmigración y mercado de trabajo en España. Informe 2012", financiado por el Ministerio de Empleo y Seguridad Social. Secretaría de Estado de Inmigración y Emigración. 
Palabras clave: cambio ocupacional, biografía laboral, trabajadores inmigrantes

Abstract: This paper aims at examining in depth the occupational change and the work trajectories of individuals through time in order to provide a wider perspective of the effects of the 2008-2013 financial crisis on employment. We use microdata from the Labour Force Survey and carry out a comparison among three groups: the Spanish born in Spain, the Spanish born abroad and the non-Spanish born abroad. The results confirm that the latter group is characterized by the existence of a segmented occupational structure towards (highskilled and low-skilled) blue-collar jobs, a fact which has fostered a loss of employment in the work biography of the youngest cohorts of individuals during the recession.

Keywords: occupational change, work biography, immigrant workers.

\section{INTRODUCCIÓN}

Los efectos de la crisis económica en España no pueden ser entendidos completamente si no se tienen en cuenta los cambios estructurales de la economía española acaecidos a lo largo de las últimas décadas que han provocado, entre otros efectos, una reubicación sectorial y ocupacional de la población, tanto de la población española de origen como de la procedente de la inmigración.

Desde el inicio de la crisis, a finales del año 2007, el mercado de trabajo ha entrado en una dinámica de cambio, en el que resaltan dos tendencias: a) cambios en la composición del empleo; y b) deterioro de la actividad laboral de los colectivos más vulnerables, especialmente jóvenes y extranjeros. Los estudios e investigaciones realizadas en los últimos años avalan dichas tendencias.

Durante el trascurso de la crisis se han producido cambios en el nivel y la composición del empleo, destacando dos aspectos: a) la destrucción de 4 millones de empleos (Banco de España, 2014a; Carrasco y García-Serrano, 2012), lo que ha supuesto un ajuste vía cantidades (finalizaciones de contratos temporales y despidos) en contraste con otras fórmulas relacionadas con la flexibilidad interna adoptadas en otros países del entorno europeo (OCDE, 2011: capítulo 1); y b) un 
comportamiento más favorable del empleo femenino (Garrido, Miyar y Muñoz, 2010; Muñoz Comet, 2012), del grupo de población de edad más avanzada y de los trabajadores con estudios universitarios (Banco de España, 2014b), lo que ha provocado una reducción de la distancia en tasas de actividad, empleo y paro entre hombres y mujeres pero ha estado asociado a una caída de la oferta laboral de los menores de 30 años (Álvarez y Davia, 2013:113). De forma complementaria, los colectivos más vulnerables coinciden, en parte, con aquellos que se encontraban en una situación de inestabilidad laboral antes del inicio de la crisis. Específicamente, la destrucción neta de puestos de trabajo durante la crisis ha afectado en mayor medida a los varones, los jóvenes menores de 30 años, la población extranjera (especialmente la no comunitaria) y las personas con nivel educativo bajo (Banco de España, 2014a).

En este contexto, el artículo tiene como objetivo profundizar en dos dimensiones relacionadas con los efectos de la crisis del empleo, en concreto los cambios en la estructura ocupacional y las alteraciones en la biografía laboral de los individuos a lo largo del tiempo. Para ello se utilizarán los microdatos de la Encuesta de Población Activa (EPA). La información se presentará referida a tres grupos de población: las personas de nacionalidad española nacidas en España, las personas de nacionalidad española nacidas en el extranjero (incluidas las que tienen doble nacionalidad) y las personas de nacionalidad extranjera nacidas fuera de España.

La estructura del artículo es la siguiente. En la sección dos, se revisa la literatura relacionada con el debate teórico más reciente sobre la integración laboral de la población inmigrada. En la sección tres, se analizan los efectos estructurales del cambio ocupacional en la población inmigrada (2000-2013). En la sección cuatro, se utiliza la metodología de las cohortes ficticias para estudiar un aspecto fundamental relacionado con la movilidad laboral, a saber, las trayectorias de empleo de los individuos a lo largo del tiempo. Finalmente, un apartado de conclusiones cierra el estudio.

\section{DEBATE TEÓRICO: ASIMILACIÓN VERSUS SEGREGACIÓN}

El debate teórico sobre la integración laboral de la población inmigrada, en concreto su inserción en la estructura ocupacional, se 
ha desarrollado en torno a dos enfoques: asimilación versus segregación.

Por un lado, el enfoque de la asimilación tiene su epicentro en la teoría del capital humano (Becker, 1975), la cual explicaría la situación de exclusión en el mercado laboral haciendo referencia a las características personales del trabajador, en especial el nivel educativo, la experiencia laboral y el tiempo de residencia en el país de acogida (en el caso de la población inmigrada). La hipótesis es que a medida que aumenta el capital humano de la población inmigrada se esperaría una asimilación con la población autóctona. Las evidencias empíricas para el caso español en torno al tiempo de residencia (Bernardi y Martínez-Pastor, 2010; Bernardi, Garrido y Miyar, 2011) no corroboran la hipótesis de la asimilación aunque encuentran grandes diferencias en atención al origen de la población inmigrante. Los resultados que avalan cierta asimilación (especialmente en tasas de empleo) se desarrollaron antes de la crisis económica o bien cuando todavía sus efectos no se habían completado (Oliver, 2011).

Por otro lado, el enfoque de la segmentación hace referencia a aspectos estructurales e institucionales (Piore, 1975), poniendo el foco de atención en la trayectoria ocupacional del individuo, de modo que el trabajador que ha estado empleado en ocupaciones no cualificadas tendría más probabilidades de quedar atrapado en este segmento secundario. El análisis de las trayectorias laborales de los inmigrantes en España utilizando datos de la Muestra Continua de Vidas Laborales (Miguélez et al., 2011) ha aportado información muy valiosa en el ámbito de la movilidad laboral avalando el enfoque de la segmentación. Entre las conclusiones principales del estudio de estos autores se pueden destacar las siguientes. En primer lugar, la gran importancia de la estructura productiva del país, que junto a aspectos de oferta (dominio del idioma, tiempo de residencia, edad, sexo y nivel de estudios), determina en gran medida la ubicación y las posibilidades de movilidad de la población inmigrante (también de la autóctona). En segundo lugar, lo que los autores denominan como «alta seguridad en el empleo a lo largo de la vida» se distribuye de forma desigual entre los colectivos, quedando reservada una seguridad más alta para los autóctonos junto con los extranjeros que proceden de la UE-15, seguidos a bastante distancia por algunos grupos procedentes de Perú, Marruecos, Ecuador y Rumanía.

El punto central del estudio consiste en el análisis de la movilidad profesional vertical, definida como los cambios de posición en 
la escala de las diez categorías profesionales construidas a partir de los grupos de cotización de la Seguridad Social². Los autores consideran movilidad ascendente baja cuando se sube 1 o 2 posiciones en la escala, movilidad ascendente media cuando se sube de 3 a 5 posiciones y movilidad ascendente alta cuando sube de 6 a 9 posiciones. Su análisis concluye que un $57 \%$ de la población asalariada autóctona registraba movilidad laboral ascendente frente a un 33\% de la población inmigrante. La imagen que más predomina en estos últimos es la del estancamiento (Miguélez et al., 2011: 335-343).

Adicionalmente, el enfoque de la segmentación ha sido contrastado, para el caso español, en referencia a la temporalidad (Martínez-Pastor, 2014), la sobrecualificación (Alonso y del Río, 2010) y el género (Del Río y Alonso-Villar, 2010), encontrando para la población inmigrada no sólo una concentración en las ocupaciones de más baja cualificación sino también en las de mayor índice de feminización/masculinización.

\section{EFECTOS ESTRUCTURALES DEL CAMBIO OCUPACIONAL EN LA POBLACIÓN INMIGRADA}

En los últimos veinticinco años, España ha vivido dos crisis económicas de una gran magnitud (desde 1991 hasta 1994 y desde 2008 hasta 2013) y una etapa de expansión muy intensa (1995-2007). Estos vaivenes han dejado su huella, entre otros aspectos, en la composición ocupacional del empleo. Para analizar su evolución, se ha procedido a utilizar los microdatos de la $\mathrm{EPA}^{3}$.

2 También se examinan la movilidad geográfica y la horizontal (que se refiere a cambios entre ocupaciones entre diferentes sectores de actividad económica).

3 La Clasificación Nacional de Ocupaciones 1994 (CNO-1994) se empleó desde 1994 hasta 2010, siendo reemplazada por la nueva Clasificación Nacional de Ocupaciones 2011 (CNO-2011) en el primer trimestre de 2011, si bien a lo largo de los cuatro trimestres de 2010 las variables de ocupación se han codificado utilizando tanto la clasificación CNO-2011 como la anterior CNO-1994. La reciente adopción de la Clasificación Nacional de Ocupaciones 2011 ha dado lugar a que, aunque se hayan mantenido los «Grandes Grupos» (que son diez) y sus denominaciones, el contenido de los mismos se ha alterado, lo que ha provocado una modificación significativa en la estructura ocupacional. A título de ejemplo, los trabajadores propietarios de la hostelería y el comercio se clasifican en el gran 
Con el fin de examinar el cambio ocupacional del empleo civil total (excluidos los trabajadores de las fuerzas armadas) en un periodo tan extenso, primeramente se han agrupado los distintos grupos de ocupaciones en cuatro grandes categorías según su naturaleza y el tipo de tareas que se llevan a cabo: las ocupaciones no manuales cualificadas ( Directivos de empresas y de la Administración Pública», «Técnicos y profesionales científicos» y «Técnicos y profesionales de apoyo»), las ocupaciones no manuales no cualificadas («Empleados administrativos» y «Trabajadores de los servicios de restauración, personales, protección y vendedores»), las ocupaciones manuales cualificadas («Trabajadores cualificados de la agricultura», «Trabajadores cualificados de industrias manufactureras y construcción» y «Operadores de instalaciones y maquinaria, y montadores») y las ocupaciones manuales no cualificadas («Trabajadores no cualificados») $)^{4}$.

Esta agregación permite comprobar los efectos de la creación y la destrucción de empleos sobre las diferentes categorías ocupacionales a medio y largo plazo así como su diferente comportamiento a lo largo del ciclo económico. Así, se detecta un incremento continuado del peso de las ocupaciones no manuales en el empleo total, de modo que dicho peso ha pasado de estar en torno al 50\% en 1994 al $62 \%$ en 2013. Al mismo tiempo, como consecuencia de que el empleo no manual permanece más o menos estable durante las etapas de crisis y crece rápidamente durante las etapas de expansión mientras que el empleo manual disminuye fuertemente en las etapas de crisis y aumenta en las etapas de expansión, la evolución de dicho peso se ve afectado por el ciclo económico: es especialmente notable el incremento de la proporción del empleo no manual al final de la recesión de los noventa (1994-1996) y, sobre todo, durante el último

grupo 1 cuando las tareas de gestión (incluida la supervisión de otros trabajadores) representen más de un tercio del tiempo de trabajo; en caso contrario se clasifican con el gran grupo correspondiente, fuera del gran grupo 1. La aplicación de esta regla supone por lo general la clasificación de estas ocupaciones fuera de éste. Esto implica una reducción del peso de este gran grupo en relación con el que tenía de acuerdo con la CNO-1994. Esta es la razón por la cual se presentan 2 tablas separadas en esta sección: la tabla 1(a) para el periodo 2000-2010 y la tabla 1(b) para el periodo 2011-2013.

4 Las primeras corresponden a los grandes grupos 1 al 3 de la CNO-1994 y de la CNO-2011, las segundas a los grandes grupos 4 y 5 , las terceras a los grandes grupos 6 al 8 y las cuartas al gran grupo 9 . 
periodo de crisis (2008-2013). Este comportamiento se explica principalmente por la evolución del empleo no manual cualificado. En cuanto al empleo manual, destaca la gran estabilidad del peso de las ocupaciones no cualificadas (lo que significa que este empleo cambia en proporciones similares al empleo total), mientras que la proporción de las cualificadas no ha dejado de reducirse como consecuencia del mantenimiento (en la expansión) y de la disminución (en la crisis) del empleo en ciertos sectores (agricultura, industria) en comparación con la evolución de la ocupación en el resto de la economía.

A continuación, se lleva a cabo de forma más desagregada un análisis del cambio ocupacional según nacionalidad y procedencia de la población. Para ello, la tabla 1(a) ofrece la distribución del empleo desagregado en los nueve grupos ocupacionales para los años 2000, 2007 y 2010 (cuartos trimestres) y la tabla 1(b) para los años 2011, 2012 y 2013.

En cuanto a los españoles nacidos en España, la consideración más desagregada del cambio ocupacional en el caso de los grupos ocupacionales que incluyen trabajos de naturaleza no manual sugiere que todos ellos han aumentado su empleo durante la expansión y apenas lo han perdido durante la recesión, lo que ha dado lugar a un gran aumento del peso de este tipo de trabajos dentro del empleo total (sobre todo entre 2007 y 2010, manteniéndose estable para el período 2011-2013). Esto es especialmente cierto en el caso de las ocupaciones cualificadas, cuyo peso alcanzaba el 35,3\% en 2013, en particular para las categorías de profesionales y técnicos científicos y de apoyo, cuyo aumento neto del empleo durante la expansión fue considerable y apenas se ha detenido durante las etapas de crisis económica y destrucción del empleo. Algo similar ha sucedido con el empleo en las ocupaciones menos cualificadas, en concreto las que recogen personas que trabajan en los servicios, cuyo empleo ha continuado creciendo durante la recesión, de forma que su peso en el empleo total se ha elevado hasta alcanzar un 32,9\% en 2013. Por lo que respecta a los grupos ocupacionales de carácter manual, tanto las ocupaciones más cualificadas como las no cualificadas mantuvieron el número de efectivos ocupados durante la expansión y lo redujeron durante la recesión, perdiendo peso en términos relativos durante todo el periodo (14 puntos porcentuales en conjunto, quedando situado en 2013 en un $31,2 \%$ ). 
En el caso del colectivo de españoles nacidos en el extranjero, se ha producido una redistribución del empleo entre las categorías de trabajos de naturaleza no manual, descendiendo el peso de las cualificadas (que pasa del 34,3\% en 2000 al 23,8\% en 2013) y aumentando el de las no cualificadas (que pasa del 26,4\% al 36,4\%). En concreto, el mayor aumento de empleo neto entre las menos cualificadas se ha producido en la categoría de los trabajadores de los servicios (hostelería, restauración, etc.). De otra parte, el peso del empleo en las categorías de naturaleza manual cualificada ha descendido en casi 6 puntos porcentuales (del 23\% en 2000 al 17,4\% en 2013), mientras que el de naturaleza manual no cualificada ha aumentado (pasando del 16,3\% en 2000 al 21,4\% en 2013). En conjunto, el peso del empleo en la categoría de naturaleza manual quedaba situado en 2013 en un $38,8 \%$.

Finalmente, en el caso de los extranjeros no nacidos en España, su estructura ocupacional se caracteriza, por comparación con los dos colectivos anteriores, por un menor peso de los puestos no manuales cualificados y uno mayor de los puestos manuales no cualificados. Sin embargo, debe señalarse que la recesión ha alterado en parte esta distribución, debido a los siguientes procesos: se ha producido un incremento de los niveles de empleo en las ocupaciones no manuales cualificadas (especialmente en la categoría de profesionales y técnicos científicos), por lo que su peso en el empleo total ha crecido (del 11,6\% en 2007 hasta el 14,1\% en 2013); la reducción del empleo en ocupaciones no manuales poco cualificadas ha sido escaso, por lo que su peso también ha aumentado (del 23,7\% en 2007 al $31 \%$ en 2013); la gran destrucción de empleo en la construcción y en la industria ha provocado una reducción importante del número de efectivos ocupados en puestos manuales cualificados, dando lugar a una disminución muy fuerte de su peso en el empleo total, que pasa del 30,5\% en 2007 al 18,7\% en 2013); y, a pesar de perder empleo, las ocupaciones manuales no cualificadas han continuado aumentando su importancia dentro de la estructura ocupacional de este colectivo, quedando situada en un $36,2 \%$ en 2013. En conjunto, el peso del empleo en la categoría de naturaleza manual era en 2013 del 54,9\%. 
TABLA $1($ A)

DISTRIBUCIÓN DEL EMPLEO POR GRUPOS DE OCUPACIÓN SEGÚN NACIONALIDAD Y PROCEDENCIA. ESPAÑA, AÑOS 2000, 2007 Y 2010 (CUARTOS TRIMESTRES)

\begin{tabular}{|c|c|c|c|c|c|c|c|c|c|}
\hline \multirow[b]{2}{*}{ NIVELES (miles) } & \multicolumn{3}{|c|}{$\begin{array}{l}\text { Españoles nacidos } \\
\text { en España }\end{array}$} & \multicolumn{3}{|c|}{$\begin{array}{c}\text { Españoles nacidos } \\
\text { fuera }\end{array}$} & \multicolumn{3}{|c|}{$\begin{array}{c}\text { Extranjeros nacidos } \\
\text { fuera }\end{array}$} \\
\hline & 2000 & 2007 & 2010 & 2000 & 2007 & 2010 & 2000 & 2007 & 2010 \\
\hline Total & 14.889 & 16.952 & 15.196 & 277 & 555 & 602 & 532 & 2.884 & 2.503 \\
\hline (1) Dirección de empresas y AA.PP. & 1.165 & 1.395 & 1.333 & 24 & 48 & 56 & 49 & 120 & 110 \\
\hline (2) Técnicos y profesionales científicos & 1.777 & 2.363 & 2.422 & 36 & 74 & 89 & 43 & 106 & 146 \\
\hline (3) Técnicos y profesionales de apoyo & 1.485 & 2.241 & 2.185 & 35 & 74 & 75 & 32 & 109 & 114 \\
\hline (4) Empleados de tipo administrativo & 1.490 & 1.737 & 1.536 & 27 & 46 & 47 & 26 & 122 & 113 \\
\hline (5) Trabajadores de servicios & 2.069 & 2.481 & 2.503 & 46 & 98 & 126 & 95 & 560 & 534 \\
\hline (6) Trab. cualificados agricultura y pesca & 676 & 461 & 401 & 2 & 5 & 5 & 11 & 38 & 49 \\
\hline (7) Trab. cualificados industria y construcción & 2.608 & 2.640 & 1.838 & 40 & 83 & 70 & 78 & 651 & 375 \\
\hline (8) Operadores instalaciones y maquinaria & 1.580 & 1.692 & 1.381 & 22 & 40 & 36 & 21 & 190 & 170 \\
\hline (9) Trabajadores no cualificados & 2.039 & 1.943 & 1.597 & 45 & 86 & 97 & 177 & 988 & 893 \\
\hline \multicolumn{10}{|l|}{ Agrupación según naturaleza } \\
\hline Total & 14.889 & 16.952 & 15.196 & 277 & 555 & 602 & 532 & 2.884 & 2.503 \\
\hline No manuales cualificados (1-3) & 4.426 & 5.999 & 5.941 & 95 & 197 & 220 & 124 & 335 & 370 \\
\hline No manuales poco cualificados (4-5) & 3.559 & 4.219 & 4.038 & 73 & 144 & 174 & 121 & 682 & 647 \\
\hline Manuales cualificados (6-8) & 4.864 & 4.792 & 3.620 & 64 & 128 & 111 & 110 & 879 & 593 \\
\hline \multirow[t]{2}{*}{ Manuales poco cualificados (9) } & 2.039 & 1.943 & 1.597 & 45 & 86 & 97 & 177 & 988 & 893 \\
\hline & \multicolumn{3}{|c|}{$\begin{array}{c}\text { Españoles nacidos en } \\
\text { España }\end{array}$} & \multicolumn{3}{|c|}{$\begin{array}{c}\text { Españoles nacidos } \\
\text { fuera }\end{array}$} & \multicolumn{3}{|c|}{$\begin{array}{c}\text { Extranjeros nacidos } \\
\text { fuera }\end{array}$} \\
\hline DISTRIBUCIÓN (\%) & 2000 & 2007 & 2010 & 2000 & 2007 & 2010 & 2000 & 2007 & 2010 \\
\hline Total & 100,0 & 100,0 & 100,0 & 100,0 & 100,0 & 100,0 & 100,0 & 100,0 & 100,0 \\
\hline (1) Dirección de empresas y AA.PP. & 7,8 & 8,2 & 8,8 & 8,9 & 8,7 & 9,3 & 9,2 & 4,1 & 4,4 \\
\hline (2) Técnicos y profesionales científicos & 11,9 & 13,9 & 15,9 & 13,0 & 13,4 & 14,8 & 8,0 & 3,7 & 5,8 \\
\hline (3) Técnicos y profesionales de apoyo & 10,0 & 13,2 & 14,4 & 12,5 & 13,4 & 12,5 & 6,0 & 3,8 & 4,6 \\
\hline (4) Empleados de tipo administrativo & 10,0 & 10,2 & 10,1 & 9,6 & 8,3 & 7,9 & 5,0 & 4,2 & 4,5 \\
\hline (5) Trabajadores de servicios & 13,9 & 14,6 & 16,5 & 16,8 & 17,7 & 21,0 & 17,8 & 19,4 & 21,3 \\
\hline (6) Trab. cualificados agricultura y pesca & 4,5 & 2,7 & 2,6 & 0,7 & 0,9 & 0,8 & 2,1 & 1,3 & 1,9 \\
\hline (7) Trab. cualificados industria y construcc. & 17,5 & 15,6 & 12,1 & 14,3 & 14,9 & 11,6 & 14,6 & 22,6 & 15,0 \\
\hline (8) Operadores instalaciones y maquinaria & 10,6 & 10,0 & 9,1 & 8,0 & 7,3 & 6,0 & 4,0 & 6,6 & 6,8 \\
\hline
\end{tabular}


TABLA 1(A)

\section{CONTINUACIÓN}

\begin{tabular}{|lc|c|c|c|c|c|c|c|c|}
\cline { 2 - 12 } \multicolumn{1}{c|}{} & \multicolumn{3}{c|}{$\begin{array}{c}\text { Españoles nacidos en } \\
\text { España }\end{array}$} & \multicolumn{3}{c|}{$\begin{array}{c}\text { Españoles nacidos } \\
\text { fuera }\end{array}$} & \multicolumn{3}{c|}{$\begin{array}{c}\text { Extranjeros nacidos } \\
\text { fuera }\end{array}$} \\
\hline DISTRIBUCIÓN (\%) & $\mathbf{2 0 0 0}$ & $\mathbf{2 0 0 7}$ & $\mathbf{2 0 1 0}$ & $\mathbf{2 0 0 0}$ & $\mathbf{2 0 0 7}$ & $\mathbf{2 0 1 0}$ & $\mathbf{2 0 0 0}$ & $\mathbf{2 0 0 7}$ & $\mathbf{2 0 1 0}$ \\
\hline (9) Trabajadores no cualificados & 13,7 & 11,5 & 10,5 & 16,3 & 15,4 & 16,2 & 33,3 & 34,3 & 35,7 \\
\hline Agrupación según naturaleza: & & & & & & & & & \\
\hline Total & 100,0 & 100,0 & 100,0 & 100,0 & 100,0 & 100,0 & 100,0 & 100,0 & 100,0 \\
\hline No manuales cualificados (1-3) & 29,7 & 35,4 & 39,1 & 34,3 & 35,4 & 36,6 & 23,2 & 11,6 & 14,8 \\
\hline No manuales poco cualificados (4-5) & 23,9 & 24,9 & 26,6 & 26,4 & 26,0 & 28,9 & 22,8 & 23,7 & 25,9 \\
\hline Manuales cualificados (6-8) & 32,7 & 28,3 & 23,8 & 23,0 & 23,1 & 18,4 & 20,6 & 30,5 & 23,7 \\
\hline Manuales poco cualificados (9) & 13,7 & 11,5 & 10,5 & 16,3 & 15,4 & 16,2 & 33,3 & 34,3 & 35,7 \\
\hline
\end{tabular}

Fuente: elaboración propia con microdatos de la EPA (INE).

TABLA 1(B)

DISTRIBUCIÓN DEL EMPLEO POR GRUPOS DE OCUPACIÓN, SEGÚN NACIONALIDAD Y PROCEDENCIA. ESPAÑA, PERIODO 2011-2013 (CUARTOS TRIMESTRES)

\begin{tabular}{|l|c|c|c|c|c|c|c|c|c|}
\cline { 2 - 13 } \multicolumn{1}{c|}{} & \multicolumn{3}{c|}{\begin{tabular}{c} 
Españoles nacidos en \\
\multicolumn{1}{c|}{}
\end{tabular}} & \multicolumn{3}{c|}{$\begin{array}{c}\text { Españoles nacidos } \\
\text { España }\end{array}$} & \multicolumn{3}{c|}{$\begin{array}{c}\text { Extranjeros nacidos } \\
\text { fuera }\end{array}$} \\
\hline NIVELES (miles) & $\mathbf{2 0 1 1}$ & $\mathbf{2 0 1 2}$ & $\mathbf{2 0 1 3}$ & $\mathbf{2 0 1 1}$ & $\mathbf{2 0 1 2}$ & $\mathbf{2 0 1 3}$ & $\mathbf{2 0 1 1}$ & $\mathbf{2 0 1 2}$ & $\mathbf{2 0 1 3}$ \\
\hline Total & 14.785 & 14.122 & 14.101 & 637 & 619 & 644 & 2.262 & 2.098 & 2.013 \\
\hline (1) Dirección de empresas y AA.PP. & 810 & 735 & 689 & 29 & 19 & 27 & 65 & 81 & 54 \\
\hline (2) Técnicos y profesionales científicos & 2.627 & 2.681 & 2.676 & 99 & 85 & 86 & 157 & 143 & 147 \\
\hline (3) Técnicos y profesionales de apoyo & 1.684 & 1.579 & 1.611 & 51 & 52 & 40 & 107 & 90 & 83 \\
\hline (4) Empleados de tipo administrativo & 1.672 & 1.583 & 1.550 & 59 & 48 & 48 & 85 & 72 & 67 \\
\hline (5) Trabajadores de servicios & 3.164 & 3.064 & 3.090 & 161 & 184 & 186 & 591 & 570 & 557 \\
\hline (6) Trab. cualificados agricultura y pesca & 408 & 401 & 392 & 9 & 9 & 10 & 40 & 38 & 34 \\
\hline (7) Trab. cualificados industria y constr. & 1.759 & 1.583 & 1.544 & 71 & 58 & 64 & 306 & 242 & 243 \\
\hline (8) Operadores instalaciones y maquinaria & 1.213 & 1.119 & 1.073 & 36 & 40 & 38 & 146 & 109 & 99 \\
\hline (9) Trabajadores no cualificados & 1.445 & 1.376 & 1.399 & 125 & 125 & 138 & 763 & 753 & 728 \\
\hline Agrupación según naturaleza & & & & & & & & & \\
\hline
\end{tabular}


TABLA 1(B)

\section{CONTINUACIÓN}

\begin{tabular}{|c|c|c|c|c|c|c|c|c|c|}
\hline \multirow[b]{2}{*}{ NIVELES (miles) } & \multicolumn{3}{|c|}{$\begin{array}{c}\text { Españoles nacidos en } \\
\text { España }\end{array}$} & \multicolumn{3}{|c|}{$\begin{array}{l}\text { Españoles nacidos } \\
\text { fuera }\end{array}$} & \multicolumn{3}{|c|}{$\begin{array}{l}\text { Extranjeros nacidos } \\
\text { fuera }\end{array}$} \\
\hline & 2011 & 2012 & 2013 & 2011 & 2012 & 2013 & 2011 & 2012 & 2013 \\
\hline Total & 14.785 & 14.122 & 14.101 & 637 & 619 & 644 & 2.262 & 2.098 & 2.013 \\
\hline No manuales cualificados (1-3) & 5.122 & 4.996 & 4.977 & 179 & 156 & 153 & 329 & 314 & 284 \\
\hline No manuales poco cualificados (4-5) & 4.835 & 4.647 & 4.640 & 219 & 232 & 234 & 676 & 641 & 624 \\
\hline Manuales cualificados (6-8) & 3.381 & 3.103 & 3.009 & 114 & 107 & 111 & 493 & 389 & 377 \\
\hline \multirow{2}{*}{ Manuales poco cualificados (9) } & 1.445 & 1.376 & 1.399 & 125 & 125 & 138 & 763 & 753 & 728 \\
\hline & \multicolumn{3}{|c|}{$\begin{array}{c}\text { Españoles nacidos en } \\
\text { España }\end{array}$} & \multicolumn{3}{|c|}{$\begin{array}{l}\text { Españoles nacidos } \\
\text { fuera }\end{array}$} & \multicolumn{3}{|c|}{$\begin{array}{l}\text { Extranjeros nacidos } \\
\text { fuera }\end{array}$} \\
\hline DISTRIBUCIÓN (\%) & 2011 & 2012 & 2013 & 2011 & 2012 & 2013 & 2011 & 2012 & 2013 \\
\hline Total & 100,0 & 100,0 & 100,0 & 100,0 & 100,0 & 100,0 & 100,0 & 100,0 & 100,0 \\
\hline (1) Dirección de empresas y AA.PP. & 5,5 & 5,2 & 4,9 & 4,6 & 3,1 & 4,2 & 2,9 & 3,9 & 2,7 \\
\hline (2) Técnicos y profesionales científicos & 17,8 & 19,0 & 19,0 & 15,5 & 13,7 & 13,4 & 6,9 & 6,8 & 7,3 \\
\hline (3) Técnicos y profesionales de apoyo & 11,4 & 11,2 & 11,4 & 8,0 & 8,4 & 6,2 & 4,7 & 4,3 & 4,1 \\
\hline (4) Empleados de tipo administrativo & 11,3 & 11,2 & 11,0 & 9,3 & 7,8 & 7,5 & 3,8 & 3,4 & 3,3 \\
\hline (5) Trabajadores de servicios & 21,4 & 21,7 & 21,9 & 25,3 & 29,7 & 28,9 & 26,1 & 27,2 & 27,7 \\
\hline (6) Trab. cualificados agricultura y pesca & 2,8 & 2,8 & 2,8 & 1,4 & 1,5 & 1,6 & 1,8 & 1,8 & 1,7 \\
\hline (7) Trab. cualificados industria y constr. & 11,9 & 11,2 & 10,9 & 11,1 & 9,4 & 9,9 & 13,5 & 11,5 & 12,1 \\
\hline (8) Operadores instalaciones y maquinaria & 8,2 & 7,9 & 7,6 & 5,7 & 6,5 & 5,9 & 6,5 & 5,2 & 4,9 \\
\hline (9) Trabajadores no cualificados & 9,8 & 9,7 & 9,9 & 19,6 & 20,2 & 21,4 & 33,7 & 35,9 & 36,2 \\
\hline \multicolumn{10}{|l|}{ Agrupación según naturaleza: } \\
\hline Total & 100,0 & 100,0 & 100,0 & 100,0 & 100,0 & 100,0 & 100,0 & 100,0 & 100,0 \\
\hline No manuales cualificados (1-3) & 34,7 & 35,4 & 35,3 & 28,1 & 25,2 & 23,8 & 14,5 & 15,0 & 14,1 \\
\hline No manuales poco cualificados (4-5) & 32,7 & 32,9 & 32,9 & 34,6 & 37,5 & 36,4 & 29,9 & 30,6 & 31,0 \\
\hline Manuales cualificados (6-8) & 22,9 & 21,9 & 21,3 & 18,2 & 17,4 & 17,4 & 21,8 & 18,5 & 18,7 \\
\hline Manuales poco cualificados (9) & 9,8 & 9,7 & 9,9 & 19,6 & 20,2 & 21,4 & 33,7 & 35,9 & 36,2 \\
\hline
\end{tabular}

Fuente: elaboración propia con microdatos de la EPA (INE).

En resumen, durante la etapa expansiva, los extranjeros nacidos fuera de España fueron engrosando las categorías ocupacionales que requerían menos cualificaciones (tanto manuales como no manuales), aunque también aumentaron su presencia en puestos 
manuales cualificados (sobre todo vinculados al sector de la construcción). La crisis económica ha hecho mella especialmente en estos últimos, lo que ha provocado un aumento del peso del resto de los grupos ocupacionales. Especialmente interesante es el hecho de que las ocupaciones no manuales cualificadas han mantenido su número de efectivos, elevando el peso que suponen dentro del empleo total de los extranjeros.

\section{ANÁLISIS DE LA MOVILIDAD LABORAL CON DATOS DE COHORTES FICTICIAS}

La información publicada periódicamente por las oficinas e institutos de estadística referida a las tasas de empleo (o de paro) por grupos de edad es de carácter transversal y, por tanto, se refieren a los individuos que tienen una determinada edad en un momento dado del tiempo. Esos datos, que son valiosos, impiden saber qué les sucede a las mismas personas a medida que van cumpliendo años. Dicho de otra forma, impiden llevar a cabo un análisis longitudinal apropiado porque no permiten seguir a los mismos individuos a lo largo de toda su vida (o de un periodo temporal suficientemente largo) de modo que sea posible construir sus trayectorias de actividad, ocupación y desempleo y compararlas con las de otros individuos nacidos con anterioridad o posterioridad.

Un método que trata de aproximarse a dicho seguimiento longitudinal es el seguimiento de cohortes ficticias. Este consiste en definir grupos de individuos (cohortes) de acuerdo con su año de nacimiento y observar cuál es su actividad, ocupación y desempleo en distintos momentos del tiempo. Este es el método que vamos a utilizar en esta sección, en el que presentamos un análisis agregado de la inmigración en España, centrándonos en el periodo 2005-2013.

\subsection{El método de cohortes ficticias}

En lugar de realizar un seguimiento de lo que les va sucediendo a los individuos aislados, el análisis agregado agrupa a los individuos en determinadas categorías que sean relevantes para el análisis. El interés de este tipo de análisis radica en que podremos comparar 
directamente a distintos colectivos y realizar de manera relativamente sencilla un análisis de su evolución a lo largo del tiempo. Para llevar a cabo un análisis de cohortes ficticias, no es necesario seguir a los mismos individuos longitudinalmente. Resulta suficiente observar una muestra representativa de personas con las mismas características a lo largo del tiempo. Por ejemplo, en el año 2005 tenemos una imagen representativa de los individuos con edades de 16-20 años, en el año 2006 tenemos una imagen representativa de los individuos con edades de 17-21 años, y así sucesivamente. Es evidente que en una encuesta como la EPA, que se renueva parcialmente, los entrevistados pertenecientes a una determinada cohorte no son los mismos en dos momentos del tiempo (en el año 2005 y en el 2013), puesto que no se entrevista a las personas de forma continuada durante ese tiempo. Pero los individuos son equivalentes desde un punto de vista estadístico, ya que al fin y al cabo la encuesta es aleatoria y está diseñada para ofrecer una representación adecuada de la población y los individuos que forman parte de la muestra en un momento dado son representativos del grupo al que pertenecen. Por tanto, un análisis de seguimiento de cohortes debería generar unos resultados muy similares a los que pudieran obtenerse de un análisis puramente longitudinal.

De acuerdo con este planteamiento, esto significa que si se definen grupos de individuos de acuerdo con su cohorte de nacimiento en un año dado de la encuesta (por ejemplo, el 2005) podría seguirse a estos grupos año a año hasta el último disponible (por ejemplo, el 2013). En particular, vamos a agrupar a los individuos según su año de nacimiento en cohortes de cinco años:

- Los nacidos entre 1985 y 1989, que tenían entre 16 y 20 años en 2005, entre 17 y 21 años en 2006, entre 18 y 22 años en 2007, y así sucesivamente.

- Los nacidos entre 1980 y 1984, que tenían entre 21 y 25 años en 2005, entre 22 y 26 años en 2006, entre 23 y 27 años en 2007, y así sucesivamente.

- Los nacidos entre 1975 y 1979, que tenían entre 26 y 30 años en 2005, entre 27 y 31 años en 2006, entre 28 y 32 años en 2007, y así sucesivamente.

El resto de las cohortes se construiría de la misma forma. El gráfico 1 permite ver de un modo sencillo cómo se organiza la información siguiendo el método de las cohortes ficticias. 


\section{GRÁFICO 1}

EJEMPLO DE CONSTRUCCIÓN DE COHORTES FICTICIAS

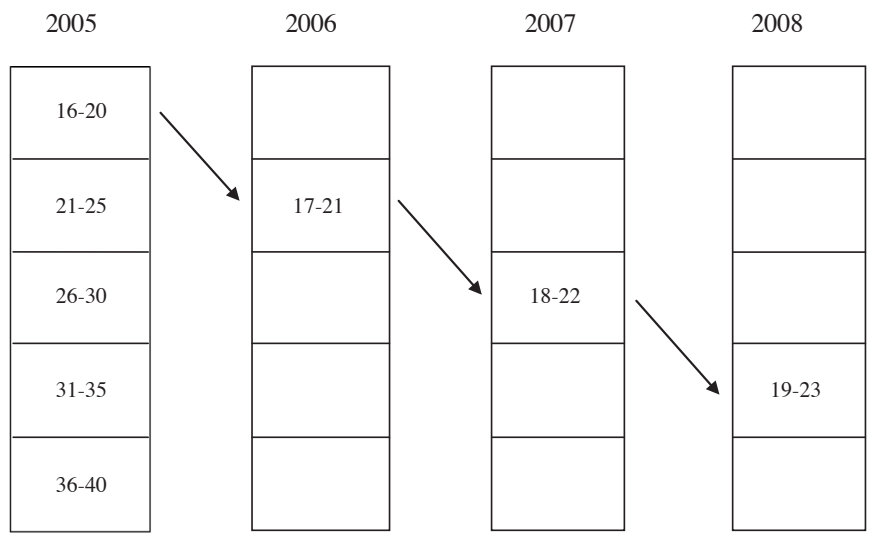

Fuente: elaboración propia.

En el caso que nos ocupa, se han construido cohortes a partir de la variable 'año de nacimiento' en cada uno de los años que van desde el 2005 hasta el 2013 utilizando explotaciones específicas de los ficheros de microdatos de la EPA. Hay que mencionar que, si bien el método no debe presentar problemas para el colectivo de españoles (nacidos en España), puesto que los que nacieron en unos determinados años son los que son sin posibilidad de adiciones posteriores, en el caso de los extranjeros no es así, sobre todo en un periodo de crecimiento continuado de las entradas. Este problema puede implicar que la representación que se realice no sea totalmente rigurosa desde el punto de vista del seguimiento longitudinal de la trayectoria de una cohorte, ya que, aunque las personas comparten el mismo periodo de nacimiento, no representan al mismo colectivo al ir cumpliendo edades sucesivas, de modo que las entradas y las salidas impedirían que se cumpliese el requisito básico del seguimiento de cohortes, ya que los individuos que tenían, por ejemplo, 36-40 años en 2010 podrían ser muy distintos de los que tenían 31-35 años en 
20055. Para minimizar el impacto de este problema, se han tomado las siguientes decisiones.

Primera, se han utilizado las cuatro EPAs trimestrales de cada año para construir ficheros anuales, de modo que la cantidad de muestra es más elevada para poder captar adecuadamente a los inmigrantes extranjeros agrupados según su año de nacimiento. Segunda, se ha elegido comenzar en el año 2005 por la misma razón. Tercera, se ha establecido la restricción de que en el primer año (2005) los extranjeros debían llevar al menos un año de residencia en España, en el segundo año (2006) al menos dos años y así sucesivamente, con objeto de intentar seguir, en la medida de lo posible, al mismo colectivo a lo largo del tiempo.

\subsection{Resultados}

En esta sección se muestran los resultados obtenidos al utilizar la metodología de las cohortes ficticias para analizar un aspecto fundamental relacionado con la movilidad laboral, a saber, las trayectorias de empleo de los individuos a lo largo del tiempo. Para ello, se ha calculado la distribución de la situación con respecto a la actividad económica del total de la población de cada una de las cohortes construidas como se ha explicado más arriba, distinguiendo las siguientes categorías: asalariados (trabajadores por cuenta ajena), otros ocupados (trabajadores por cuenta propia: empresarios, autónomos, cooperativistas y ayudas familiares), parados (personas sin empleo pero buscándolo activamente y disponibles para trabajar) e inactivos (personas sin empleo que no buscan y que no están disponibles). Esta distribución se ha obtenido para todos los años desde el 2005 hasta el 2013 y a partir de dicha distribución se ha calculado la tasa de empleo como el número de ocupados en cada grupo en relación con la población total de cada grupo. Los gráficos 2, 3 y 4 ofrecen los resultados de este ejercicio, representando las tasas de empleo para los tres colectivos de nuestro estudio: españoles

5 Este problema también puede aparecer cuando se analiza un colectivo concreto definido mediante una variable general (como la actividad laboral o ser asalariado) dentro de la población total. Para el caso particular de los asalariados y la evolución de las trayectorias de temporalidad utilizando el método de las cohortes ficticias, véase Garrido y Gutiérrez (2011). 
nacidos en España, españoles nacidos fuera de España y extranjeros nacidos fuera de España.

Los datos de los tres gráficos ofrecen una apariencia similar a la que se obtiene cuando se representan las tasas de empleo por edades correspondientes a un determinado año (corte transversal): una relación en forma de $\mathrm{U}$ invertida entre las tasas de empleo y la edad, de modo que dichas tasas crecen rápidamente hasta los 30 años, se mantienen en niveles cercanos al $80 \%$ hasta casi los 50 años y a partir de entonces comienzan a disminuir. Esta forma de campana se observa más claramente para los españoles nacidos en España que para los otros dos colectivos, pero para estos últimos también es muy aparente si se toman como puntos de observación los correspondientes a los tres primeros años (2005-2007) de cada cohorte. La diferencia más relevante radica en que las tasas de empleo de los más jóvenes comienzan en niveles más elevados (unos diez puntos porcentuales) entre los extranjeros nacidos fuera de España que en los otros dos colectivos.

\section{GRÁFICO 2}

TASAS DE EMPLEO DE LAS COHORTES DE NACIMIENTO EN EL PERIODO 2005-2013: ESPAÑOLES NACIDOS EN ESPAÑA

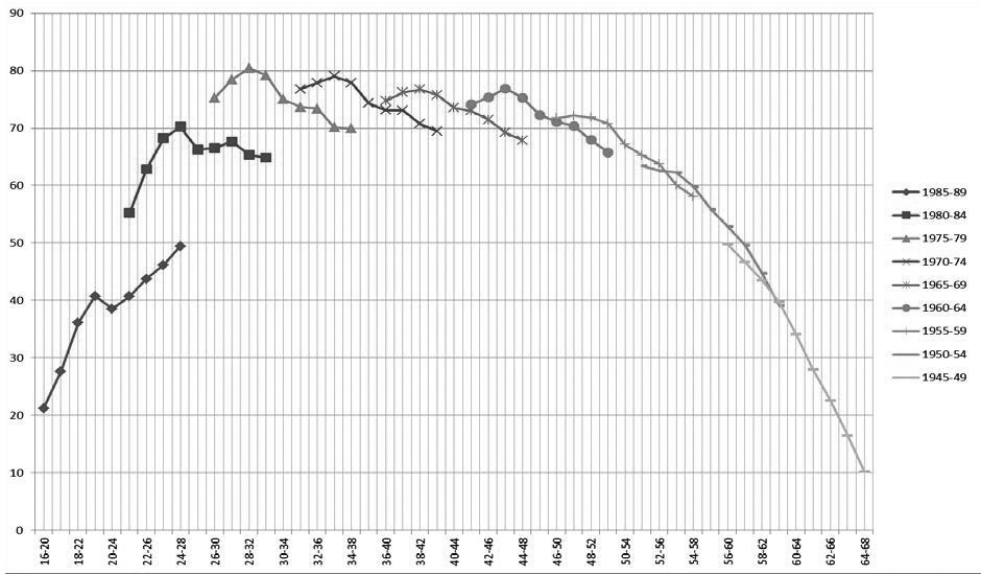

Fuente: elaboración propia con microdatos de la EPA (INE). 


\section{GRÁFICO 3}

TASAS DE EMPLEO DE LAS COHORTES DE NACIMIENTO EN EL PERIODO 2005-2013: ESPAÑOLES NACIDOS FUERA DE ESPAÑA

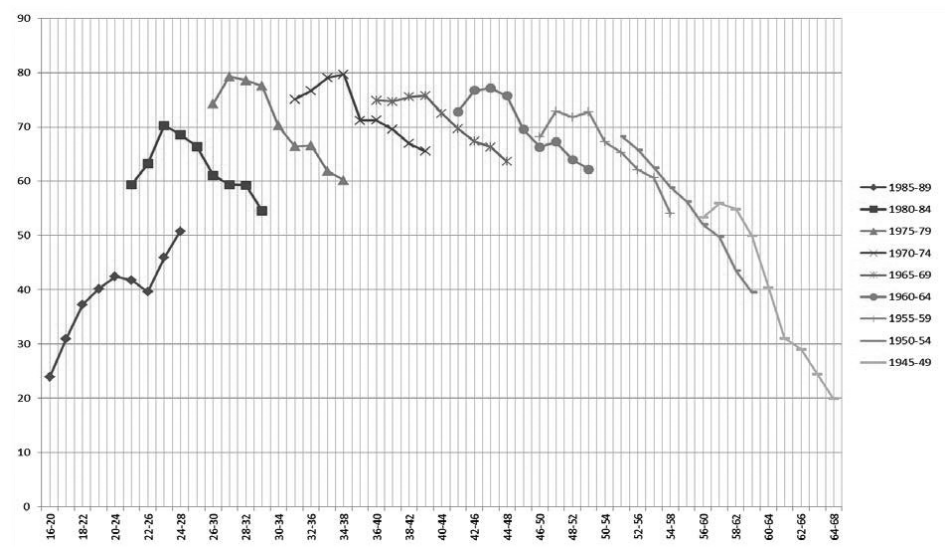

Fuente: elaboración propia con microdatos de la EPA (INE).

\section{GRÁFICO 4}

TASAS DE EMPLEO DE LAS COHORTES DE NACIMIENTO EN EL PERIODO 2005-2013: EXTRANJEROS NACIDOS FUERA DE ESPAÑA (CONTROLANDO EL NÚMERO DE AÑOS DE RESIDENCIA)

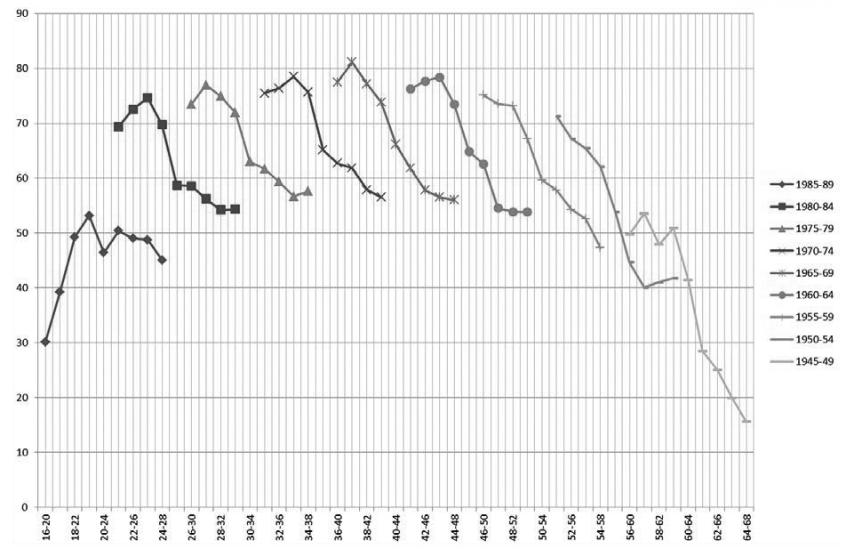

Fuente: elaboración propia con microdatos de la EPA (INE). 
Lo interesante del análisis de cohortes es que permite no sólo examinar lo que les ha sucedido en el pasado (en este caso, en el periodo 2005-2013) a los mismos individuos seguidos longitudinalmente sino también llevar a cabo una aproximación a lo que podría haber sucedido si se hubieran mantenido las circunstancias económicas prevalentes en el periodo 2005-2007 a lo largo del tiempo. En efecto, si la expansión económica hubiese continuado y los individuos no hubieran cambiado su comportamiento con respecto al mercado de trabajo, lo que cabría esperar es que la relación entre tasas de empleo y edad hubiese mantenido la forma de campana antes descrita de un modo más o menos suavizado.

De este modo, por ejemplo, la cohorte de españoles nacidos en España en 1985-1989 mostraba tasas de empleo del 21\% en 2005 (cuando tenían 16-20 años) y del 36\% en 2007 (cuando tenían 18-22 años). Si todo hubiese continuado igual, estos individuos deberían haber alcanzado tasas de empleo en torno al 55\% al cumplir los 21-25 años (como la cohorte nacida en 1980-1984 con esa edad en 2005) y alrededor del 68\% al cumplir los 23-27 años (como la cohorte anterior con esa edad en 2007).

Por tanto, la distancia vertical entre las últimas cuatro observaciones de cada cohorte y las cuatro primeras de la siguiente (observaciones que corresponden a momentos distintos [2010-2013 y 2005-2008, respectivamente] en que ambas tienen las mismas edades) podrían tomarse por una aproximación a la "pérdida de ocupación" provocada por la crisis de empleo en la biografía de los individuos que forman parte de cada cohorte con respecto a la trayectoria que deberían haber seguido si las condiciones económicas no se hubiesen modificado.

En el caso de los españoles nacidos en España, esta distancia es mayor para los más jóvenes y además se ha agrandado con la recesión: en 2010 era de unos 15 puntos porcentuales para los que tenían 16-20 años en 2005 y unos 8 puntos porcentuales para los que tenían 21-25 años en 2005, pero ha aumentado en 2012-2013 a unos 20 puntos porcentuales para los primeros y 15 puntos porcentuales para los segundos. Dicha distancia, que apenas existía para las personas de 35-50 años hasta 2010, se ha hecho positiva (en torno a 5 puntos porcentuales). Finalmente, la diferencia es prácticamente nula para los mayores de 50 años (la tasa de empleo de una cohorte es aproximadamente igual que la de la siguiente en los años en que se solapan). 
Estas distancias son mayores en el colectivo de españoles nacidos fuera de España pero sobre todo en el de los extranjeros nacidos fuera de España, en cuyo caso las diferencias superaban los 10 puntos porcentuales en 2010 y se situaban en los 15-20 puntos porcentuales en 2012-2013 en todas las cohortes (salvo las dos últimas). Esto significa que la crisis de empleo ha azotado sin excepción a todos los grupos de extranjeros independientemente de su edad, aunque la intensidad fue mayor entre los más jóvenes y menor entre los de más edad.

El caso de los más jóvenes merece una atención especial. La utilización de datos longitudinales, que permiten aproximarse a la trayectoria laboral a lo largo del ciclo vital de los individuos, aporta una visión algo diferente de la que ofrecen los datos transversales de tasas de empleo (o de paro) por grupos de edad. Como puede verse en los datos presentados en los gráficos, las tasas de empleo de la cohorte de los más jóvenes (16-20 años en 2005) de los españoles nacidos en España no dejan de crecer a lo largo de los nueve años de análisis (excepto en 2009), aunque como se ha dicho antes este aumento no se produce al mismo ritmo al que se habría producido si las condiciones económicas no se hubiesen modificado drásticamente a partir de 2008. Aun así, la tasa de empleo de este grupo, que era del 21\% en 2005 (cuando tenían 16-20 años), se situaba en el 50\% en 2013 (cuando dichos individuos tenían 24-28 años).

El caso de los españoles nacidos fuera de España es muy similar al que se acaba de comentar (la diferencia es que la reducción de la tasa de ocupación se produce en 2010-2011 en vez de 2009). Sin embargo, el de los extranjeros nacidos fuera de España es claramente distinto, ya que su tasa de empleo no solo no aumenta sino que muestra una ligera tendencia decreciente a partir de 2008, de modo que en 2013 es menor (un 45\%) en comparación con 2008 (un 53\%). Por tanto, este colectivo de jóvenes es el que ha sido golpeado en mayor medida por la crisis, ya que sus trayectorias laborales han empeorado sustancialmente con el largo periodo de destrucción de empleo que se inició en 2008.

Con respecto a la siguiente cohorte de jóvenes (21-25 años en 2005), entre los españoles nacidos en España las tasas de ocupación del periodo 2009-2013 no han variado mucho, aunque se han situado en niveles algo inferiores al máximo alcanzado por dicha cohorte en 2008. Sin embargo, entre los españoles nacidos fuera de España y, sobre todo, entre los extranjeros, la caída de las tasas de 
ocupación de esta cohorte ha sido enorme, situándose dichas tasas en torno al 55\% en 2012-2013, es decir, en niveles inferiores a los que tenía la cohorte en 2005 (especialmente en el caso de los extranjeros) y solo ligeramente superiores a las del grupo de los más jóvenes.

\section{CONCLUSIONES}

Los cambios ocupacionales en la economía española durante los últimos veinticinco años han generado una estructura segmentada para la población extranjera nacida fuera de España hacia ocupaciones de naturaleza manual tanto cualificadas (construcción e industria, fundamentalmente) como no cualificadas. Esta situación estructural ha sido el cimiento sobre el que se ha producido la gran destrucción del empleo durante la crisis económica de 2008-2013. Esto ha provocado una reducción importante del número de efectivos ocupados en puestos manuales cualificados, dando lugar a una disminución muy fuerte de su peso en el empleo total; a pesar de perder empleo, las ocupaciones manuales no cualificadas han continuado aumentando su importancia dentro de la estructura ocupacional de este colectivo.

Por otra parte, el análisis de la movilidad laboral utilizando la metodología de las cohortes ficticias ha permitido extraer al menos dos resultados de interés. El primero es que, en términos generales, las trayectorias de empleo de los extranjeros (medidas a través de las tasas de empleo de los mismos individuos a lo largo del tiempo) no parecen diferir sustancialmente de las observadas en el caso de los españoles cuando se considera el ciclo vital completo. El segundo es que el cambio de las condiciones económicas a partir del año 2008, que perduran hasta 2013, ha golpeado especialmente a la población extranjera, lo que ha dado lugar a una pérdida de ocupación en la biografía de los individuos (en términos de reducción de sus tasas de ocupación con respecto a las que tuvieron individuos de cohortes anteriores con su misma edad) que tardará años en recuperarse. El impacto de la crisis de empleo ha sido más duro en el caso de las cohortes de edad más jóvenes, cuya integración y consolidación en el mercado laboral se ha visto severamente afectada. 


\section{REFERENCIAS BIBLIOGRÁFICAS}

Alonso, O. y del Río, C. (2010): Occupational segregation of immigrants in Spain. Madrid, Instituto de Estudios Fiscales.

Álvarez, C. y Davia, M.A. (2013): «El deterioro del mercado de trabajo tras cinco años de crisis: efectos laborales y sociales». Documentación Social, 166, pp. 109-126.

BANCO DE ESPAÑA, (2014a): La evolución del empleo y del paro en 2013, según la Encuesta de Población Activa. Boletín Económico, febrero, n ${ }^{\circ}$ 41. Disponible en: http://www.bde.es [Consulta: 5/06/2014].

- (2014b). El empleo en la UEM en 2013. Boletín Económico, abril, no 84. Disponible en: http://www.bde.es [Consulta: 5/06/2014].

Becker, G. (1975): Human Capital: A Theoretical and Empirical Analysis, with Special Reference to Education. National Bureau of Economic Research.

Bernardi, F. y Martínez-PAstor, J.I. (2010): «Falling at the bottom: Unskilled jobs at entry in the Labour Market in Spain over time and in a comparative perspective». International Journal of Comparative Sociology, 51, pp. 289-307.

Bernardi, F.; Garrido, L. y Miyar, M. (2011): «The recent fast upsurge of immigrants in Spain and their employment patterns and occupational attainment». International Migration, 49 (1), pp. 148-187.

Carrasco, C. y García-SERrano, C. (2012): Inmigración y mercado de trabajo. Informe 2011. Documentos del Observatorio Permanente de la Inmigración, 28. Madrid, Ministerio de Empleo y Seguridad Social.

DEL Río, C. y Alonso-VIllaR, O. (2010): Occupational segregation of immigrant women in Spain. Working paper 165, Society for the Study of Economic Inequality.

GARrido, L. y GutiÉRrez, R. (2011): «La reforma ineludible. Regularidades e inercias del mercado de trabajo en España». Panorama Social, 13, pp. 37-54.

Garrido, L.; Miyar, M. y Muñoz, J. (2010): «La dinámica laboral de los inmigrantes en el cambio de fase del ciclo económico». Presupuesto y Gasto Público, 6, pp. 201-221.

Martínez-Pastor, J.I. (2014): «Fixed-term contracts: does nationality matter?» Journal of Ethnic and Migration Studies, 40 (5), pp. 814-828.

Miguélez, F.; Martín, A.; De Alós-Moner, R.; Esteban, F.; López Roldán, P.; Molina, O. y Moreno, S. (2011): Trayectorias laborales de los inmigrantes en España. Barcelona, Obra Social «la Caixa».

Muñoz Comet, J. (2012): «Evolución del empleo y del paro de las mujeres inmigrantes en el mercado de trabajo español. El impacto de la actual 
crisis económica». Cuadernos de Relaciones Laborales, Oso, L. y S. Parella (coord.), 30, 1, pp. 115-137.

OCDE (2011): OECD Employment Outlook. OECD Publishing.

Oliver, J. (2011): «El mercado de trabajo de la inmigración 2007-2010. Los cambios 2009/2010 en el marco de la crisis». En Inmigración y crisis económica Aja, E.; Arango, J. y Oliver, J. (dir.). Barcelona, Anuario de la Inmigración en España. CIDOB, Diputación de Barcelona, Fundación ACSAR, Fundación Ortega y Gasset, Junta de Andalucía y Unicaja, pp. $130-164$.

Piore, M. (1975): «Notas para una teoría de la estratificación del mercado de trabajo", en El mercado de trabajo. Teorías y aplicaciones, compilado por L. Toharia, 1983, Madrid, Alianza Editorial, pp. 193-222. 